\title{
Secukinumab: A Review in Moderate to Severe Pediatric Plaque Psoriasis
}

\author{
Hannah A. Blair ${ }^{1}$ \\ Accepted: 3 October 2021 / Published online: 19 October 2021 \\ C) Springer Nature 2021, corrected publication 2021
}

\begin{abstract}

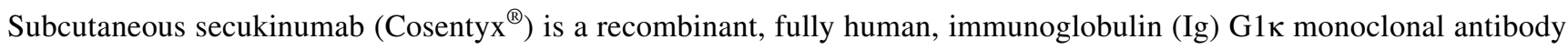
targeted against interleukin (IL)-17A, a proinflammatory cytokine involved in the pathogenesis of psoriasis. Secukinumab is approved in the EU and the USA for the treatment of moderate to severe plaque psoriasis in pediatric patients aged $\geq 6$ years. In pivotal phase III trials in pediatric patients aged 6 to $<18$ years, both low $(75-150 \mathrm{mg})$ and high $(75-300 \mathrm{mg})$ doses of secukinumab were significantly better than placebo and numerically better than etanercept at week 12 in terms of the proportion of patients achieving $\geq 75 \%$ improvement from baseline in Psoriasis Area and Severity Index and significantly better than placebo and etanercept in terms of the proportion of patients achieving an Investigator's Global Assessment score of 0 or 1 . The clinical efficacy of secukinumab observed during the first 12 weeks of treatment was maintained over the longer term. Treatment with secukinumab improved health-related quality of life and was generally well tolerated. In conclusion, secukinumab represents a valuable new addition to the limited treatment options available for children and adolescents with moderate to severe plaque psoriasis.
\end{abstract}

\section{Plain Language Summary}

Plaque psoriasis is a chronic, inflammatory skin condition that can have a negative impact on the quality of life of affected children and their families. Compared with the expanding treatment options for adults with plaque psoriasis, the number of approved medications for pediatric plaque psoriasis is relatively low. Subcutaneous secukinumab (Cosentyx $\left.{ }^{\circledR}\right)$ is one of several targeted biologic agents that have recently been approved for treating plaque psoriasis in pediatric patients. Secukinumab binds to IL-17A and inhibits the release of proinflammatory cytokines and chemokines. Treatment with secukinumab provided fast and durable skin clearance and continuous improvements in health-related quality of life in children and adolescents aged 6 to $<18$ years with moderate to severe plaque psoriasis. The benefits of secukinumab were maintained over the longer term and the drug was generally well tolerated. With a convenient 4-weekly maintenance dosing regimen and the option of caregiver administration, secukinumab is a valuable option for the treatment of moderate to severe pediatric plaque psoriasis.

Digital Features for this Adis Drug Evaluation can be found at https://doi.org/10.6084/m9.figshare.16727383.

The manuscript was reviewed by: $A$. Reich, Department of Dermatology, Institute of Medical Sciences, Medical College of Rzeszow University, Rzeszow, Poland; T. Torres, Department of Dermatology, Centro Hospitalar Universitario do Porto, Porto, Portugal.

Hannah A. Blair

demail@springer.com

1 Springer Nature, Private Bag 65901, Mairangi Bay, Auckland 0754, New Zealand

\section{Introduction}

Plaque psoriasis is a chronic, immune-mediated, inflammatory disease $[1,2]$ affecting approximately $1 \%$ of children [3-5]. It is often associated with metabolic, cardiovascular, gastrointestinal, and psychological comorbidities $[1,4]$, and can have a significant negative impact on quality of life (QOL) [3-5]. In pediatric patients, the impact of psoriasis on QOL is estimated to be greater than that of diabetes or epilepsy and comparable to that of asthma or arthritis [6].

Although many effective and well-tolerated therapies are available for the treatment of plaque psoriasis in adults [1], treatment options for children and adolescents with plaque 
Secukinumab: clinical considerations in moderate to severe pediatric plaque psoriasis

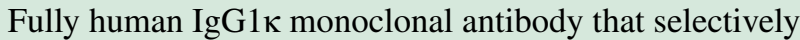
targets IL-17A

Provides fast and durable skin clearance in children and adolescents aged 6 to $<18$ years

Beneficial effects maintained over the longer term

Improves health-related quality of life

Generally well tolerated

psoriasis are much more limited $[1,2,4,5]$. This is due, at least in part, to the unpredictability of adult-approved treatments in the pediatric population [1]. Recently, several targeted biologic agents have been approved for the treatment of pediatric plaque psoriasis, including the tumor necrosis factor- $\alpha$ inhibitors etanercept and adalimumab (EU only), the interleukin (IL)-12/23 inhibitor ustekinumab, and the IL-17 inhibitors ixekizumab and secukinumab [1].

IL-17 is a key proinflammatory cytokine implicated in the pathogenesis of psoriasis [7]. IL-17A (a member of the IL-17 family) is upregulated in psoriatic lesional and non-lesional skin [7]. Secukinumab (Cosenty $\mathrm{x}^{\circledR}$ ), an IL17A antagonist, is approved in the EU [8] and the USA [9] for the treatment of moderate to severe plaque psoriasis in children and adolescents aged $\geq 6$ years. The pharmacological properties of secukinumab have been reviewed in detail previously and are summarized in Table 1 . This review focuses on the clinical use of secukinumab in pediatric plaque psoriasis. Discussion of the use of secukinumab in other approved indications (i.e. adult plaque psoriasis [10], psoriatic arthritis [11, 12], ankylosing spondylitis [13, 14], and non-radiographic axial spondyloarthritis) is outside the scope of this article.

\section{Therapeutic Efficacy of Secukinumab}

The efficacy of secukinumab for the treatment of pediatric plaque psoriasis was evaluated in two phase III trials: a randomized, double-blind, multicentre, placebo- and active comparator-controlled trial in patients with severe plaque psoriasis [15] and an open-label, multicentre trial in patients with moderate to severe plaque psoriasis [16].

\subsection{Severe Plaque Psoriasis}

The first trial enrolled pediatric patients aged 6 to $<18$ years with severe chronic plaque psoriasis for $\geq 3$ months who were candidates for systemic therapy [15]. All patients had a Psoriasis Area Severity Index (PASI) score of $\geq 20$, an Investigator's Global Assessment modified 2011 (IGA) score of 4 , and body surface area (BSA) involvement of $\geq 10 \%$. The mean age of patients at baseline was 13.5 years and most $(77.2 \%)$ patients were aged $\geq 12$ years. The mean total BSA affected by plaque psoriasis was $40 \%$ and the mean duration of plaque psoriasis was 5.22 years. Overall, $8.6 \%$ of patients had a diagnosis of psoriatic arthritis at baseline [15].

Patients were randomized to receive low-dose secukinumab (75 mg in patients weighing $<50 \mathrm{~kg}$ and $150 \mathrm{mg}$ in patients weighing $\geq 50 \mathrm{~kg}$ ), high-dose secukinumab (75 mg in patients weighing $<25 \mathrm{~kg}, 150 \mathrm{mg}$ in patients weighing 25 to $<50 \mathrm{~kg}$, and $300 \mathrm{mg}$ in patients weighing $\geq 50 \mathrm{~kg}$ ), subcutaneous etanercept $0.8 \mathrm{mg} / \mathrm{kg}$ (up to a maximum of $50 \mathrm{mg}$ ), or placebo [15]. Secukinumab and placebo were administered at weeks $0,1,2,3$, and 4 , then every 4 weeks, while etanercept was administered once weekly. The trial included a 12-week placebo- and active comparator-controlled induction period, followed by an active comparatorcontrolled maintenance period (week 12 to week 52), an extension treatment period (week 52 until week 236), and a 16-week treatment-free follow-up period. At week 12, patients in the placebo group who were classified as nonresponders were switched to either low- or high-dose secukinumab; placebo responders were discontinued from the trial. At week 52, patients in the etanercept group discontinued treatment and entered the treatment-free follow-up period, while all secukinumab recipients entered the extension treatment period, which is ongoing [15].

The co-primary endpoints were the proportion of patients achieving $\geq 75 \%$ improvement in the PASI score (PASI 75) and the proportion of patients achieving an IGA score of 0 or 1 (IGA 0/1) at week 12 for secukinumab compared with placebo [15]. The key secondary endpoint was superiority of secukinumab versus placebo for the proportion of patients achieving PASI 90 at week 12. The efficacy of secukinumab versus etanercept for the proportions of patients achieving PASI 75/90/100 or IGA 0/1 was evaluated as an exploratory objective [15].

\subsubsection{Clinical Response}

Secukinumab was efficacious for the treatment of severe plaque psoriasis in pediatric patients [15]. The clinical efficacy of secukinumab was seen as early as week 4 . At week 12, PASI 75, IGA 0/1, and PASI 90 response rates were significantly higher with both low- and highdose secukinumab than with placebo (Table 2). Low- and high-dose secukinumab were associated with significantly higher IGA 0/1 and PASI 90 response rates, and numerically higher PASI 75 and PASI 100 response rates, than etanercept at week 12 (Table 2). Among patients weighing 
Table 1 Overview of key pharmacologic properties of secukinumab $[8,9]$

Mechanism of action

Pharmacodynamics
Recombinant, fully human IgG1к monoclonal antibody

Binds selectively to and neutralizes IL-17A, thereby inhibiting its interaction with the IL-17 receptor; inhibits the release of proinflammatory cytokines and chemokines

Reduces IL-17A-mediated contributions to autoimmune and inflammatory diseases

Initial $\uparrow$ in serum levels of total IL-17A (free + SEC-bound IL-17A), followed by slow $\downarrow$ due to reduced clearance of SEC-bound IL-17A

Clinically relevant levels of SEC reach the skin, resulting in $\downarrow$ local inflammatory markers and $\downarrow$ erythema, induration, and desquamation in PP lesions

$\downarrow$ Epidermal neutrophils and neutrophil-associated markers in lesional skin of PP pts after 1-2 wks of treatment

$\downarrow$ Levels of C-reactive protein (marker of inflammation) within 1-2 wks of treatment

\section{Pharmacokinetics $^{\mathrm{a}}$}

Dose-proportional pharmacokinetics over dose range of $25-300 \mathrm{mg} ; \mathrm{C}_{\max }$ reached $\approx 6$ days following single dose $(150 \mathrm{or} 300 \mathrm{mg})$; steady-state concentrations reached after 20-24 wks of monthly administration; bioavailability $60-77 \%$

Mean trough concentrations at wks 4 and 12 were $\uparrow 23-30 \%$ after administration via Sensoready ${ }^{\circledR}$ pen than after administration of lyophilized powder and $\uparrow 23-26 \%$ than after administration via prefilled syringe

Steady-state mean trough concentrations at wk 24 were $32.6 \mu \mathrm{g} / \mathrm{mL}$ in pediatric pts weighing $<25 \mathrm{~kg}$ receiving SEC $75 \mathrm{mg}, 19.8 \mu \mathrm{g} / \mathrm{mL}$ in pts weighing $25-49 \mathrm{~kg}$ receiving SEC $75 \mathrm{mg}$, and $27.3 \mu \mathrm{g} / \mathrm{mL}$ in pts weighing $\geq 50 \mathrm{~kg}$ receiving SEC $150 \mathrm{mg}$

Vd during terminal phase 7.10-8.60 L (following single IV dose); SEC concentrations in interstitial fluid in lesional and non-lesional skin were

$27-40 \%$ of those in serum following single $300 \mathrm{mg}$ dose

Most elimination of IgG occurs via intracellular catabolism (following endocytosis); systemic clearance $\approx 0.19 \mathrm{~L} /$ day; mean half-life 22-31 days

Special populations ${ }^{\mathrm{b}}$

$\uparrow$ SEC clearance and Vd with $\uparrow$ bodyweight

Hepatic impairment and abnormal kidney function are not expected to influence SEC clearance (lack of data)

Drug interactions ${ }^{\mathrm{b}}$

Formation of CYP enzymes can be altered by $\uparrow$ levels of certain cytokines during chronic inflammation

No interaction when SEC is coadministered with methotrexate \pm corticosteroids or with midazolam (CYP3A4 substrate)

Consider monitoring and dosage adjustment when initiating or discontinuing SEC in pts receiving concomitant CYP450 substrates (particularly those with narrow therapeutic index)

$\downarrow$ decrease(d), $\uparrow$ increase(d), $C_{\max }$ maximum plasma concentration, $I g$ immunoglobulin, $I L$ interleukin, $I V$ intravenous, $P P$ plaque psoriasis, $p t s$ patients, $S E C$ secukinumab, $V d$ volume of distribution, $w k(s)$ week(s)

${ }^{a}$ All pharmacokinetic parameters are for subcutaneous SEC in pts with PP unless otherwise stated

${ }^{\mathrm{b}}$ Consult local prescribing information for detailed recommendations

$\geq 25$ to $<50 \mathrm{~kg}$, PASI 75/90/100 and IGA 0/1 response rates at week 12 were numerically higher with high-dose secukinumab $(n=15)$ than with low-dose secukinumab $(n=17)$ [15]. At week 12, mean PASI scores decreased (improved) from baseline by $82.9 \%$ in the low-dose secukinumab group and $79.9 \%$ in the high-dose secukinumab group, compared with $29.3 \%$ in the placebo group and $74.2 \%$ in the etanercept group [15].

The clinical responses reported with secukinumab at week 12 were maintained over the longer term $[15,17]$. PASI response rates reached a peak between weeks 20 and 28 and were sustained through week 52 [15] and week 104 [17] in both secukinumab groups. Similarly, IGA 0/1 response rates peaked at week 24 and were sustained through week 52 [15] and week 104 [17] in both secukinumab groups. At week 52, PASI 75 response rates in the low- and high-dose secukinumab groups were numerically higher than those in the etanercept group (87.5 and 87.5 vs $68.3 \%$, respectively) [15]. Similar results were seen with regard to IGA 0/1 (72.5 and 75.0 vs $56.1 \%$ ), PASI 90 (75.0 and 80.0 vs $51.2 \%$ ), and PASI 100 (40.0 and 47.5 vs $22.0 \%$ ) response rates. Among patients weighing $\geq 50 \mathrm{~kg}$, PASI 75/90/100 and IGA 0/1 response rates at week 52 were numerically higher with high-dose secukinumab $(n=21)$ than with low-dose secukinumab $(n=22)$. However, in the $\geq 25$ to $<50 \mathrm{~kg}$ subgroup, PASI and IGA response rates at week 52 were numerically higher with low- $(n=17)$ versus high-dose $(n=15)$ secukinumab [15].

Among patients initially randomized to placebo who switched to secukinumab at week 12 , PASI 75/90/100 and IGA 0/1 response rates at week 52 were similar to those in patients initially randomized to secukinumab [15]. 
Table 2 Efficacy of secukinumab at week 12 in pediatric patients aged 6 to $<18$ years with moderate to severe plaque psoriasis in pivotal phase III trials

\begin{tabular}{|c|c|c|c|c|c|c|}
\hline \multirow[t]{2}{*}{ Trial } & \multirow[t]{2}{*}{ Treatment (no. of pts) } & \multicolumn{3}{|c|}{ PASI response (\% of pts) } & \multirow[t]{2}{*}{ IGA $0 / 1(\% \text { of pts })^{\mathrm{a}}$} & \multirow{2}{*}{$\begin{array}{l}\text { CDLQI } 0 / 1 \\
\text { (\% of pts) }\end{array}$} \\
\hline & & PASI $75^{a}$ & PASI $90^{b}$ & PASI 100 & & \\
\hline \multirow[t]{4}{*}{ Severe psoriasis [15] } & SEC LD (40) & $80.0^{* *}$ & $72.5 * * \dagger$ & 30.0 & $70.0^{* * \dagger}$ & $44.7 *$ \\
\hline & SEC HD (40) & $77.5^{* *}$ & $67.5 * * \dagger$ & 27.5 & $60.0^{* * \dagger}$ & $50.0 *$ \\
\hline & ETA (41) & 63.4 & 29.3 & 17.1 & 34.1 & 36.6 \\
\hline & PL (41) & 14.6 & 2.4 & 0.0 & 4.9 & 15.0 \\
\hline \multirow{2}{*}{$\begin{array}{r}\text { Moderate to severe } \\
\text { psoriasis }[16,18]\end{array}$} & SEC LD (42) & 92.9 & 69.0 & 59.5 & 78.6 & 50.0 \\
\hline & SEC HD (42) & 92.9 & 76.2 & 54.8 & 83.3 & 61.9 \\
\hline
\end{tabular}

Efficacy analyses were conducted in the full analysis set

$C D L Q I$ Children's Dermatology Life Quality Index, ETA etanercept, $H D$ high dose, IGA Investigator's Global Assessment modified 2011, $L D$ low dose, PASI Psoriasis Area and Severity Index, PASI $x$ improvement of $\geq \mathrm{x} \%$ from baseline in PASI score, $P L$ placebo, pts patients, SEC secukinumab

$* p<0.01, * * p<0.0001$ vs PL; ${ }^{\dagger} p<0.05$ vs ETA

${ }^{a}$ Co-primary endpoint for SEC vs PL (historical [16])

${ }^{\mathrm{b}}$ Key secondary endpoint for SEC vs PL (historical [16])

\subsubsection{Health-Related Quality of Life}

Secukinumab improved health-related QOL (HR-QOL) in pediatric patients with severe plaque psoriasis [15]. At week 12, the proportion of patients achieving a Children's Dermatology Life Quality Index (CDLQI) score of 0 or 1 (i.e. no impact on QOL) was significantly higher with lowand high-dose secukinumab versus placebo and numerically higher versus etanercept (Table 2). The proportion of patients with a CDLQI score of 0 or 1 at week 52 was $60.6 \%$ in the low-dose secukinumab group, $66.7 \%$ in the high-dose secukinumab group and $44.4 \%$ in the etanercept group [15]. At week 104, the proportion of patients with a CDLQI score of 0 or 1 was $61.1 \%$ with any low dose of secukinumab and $65.0 \%$ with any high dose of secukinumab [17].

At week 12, the mean change from baseline in CDLQI total score (scores range from 0 to 30 [18]) was -9.05 with low-dose secukinumab, -7.71 with high-dose secukinumab, -3.79 with placebo, and -6.49 with etanercept [15]. The mean change from baseline in CDLQI total score at week 52 was - 9.2 with low-dose secukinumab, -8.3 with high-dose secukinumab, and -5.7 with etanercept. At weeks 12 and 52, improvements from baseline in CDLQI individual domain scores (symptoms and feelings, personal relationships, leisure, school or holidays, sleep, and treatment) were numerically higher in both secukinumab groups than in the etanercept group, with the exception of the personal relationship score which was numerically higher with etanercept than secukinumab at week 12 [15].

\subsection{Moderate to Severe Plaque Psoriasis}

The second trial enrolled patients aged 6 to $<18$ years with moderate to severe plaque psoriasis for $\geq 3$ months who were eligible for systemic therapy [16]. They had a PASI score of $>12$, an IGA score of $>3$, and BSA involvement of $>10 \%$. At baseline, $72.6 \%$ of patients had moderate disease and $27.4 \%$ had severe disease. The mean age of patients was 12.6 years and $60.7 \%$ of patients were 12 to $<18$ years of age. The mean total BSA involvement was $30 \%$. Patients were randomized (with stratification by bodyweight and disease severity) to receive low-dose secukinumab $(75 \mathrm{mg}$ in patients weighing $<50 \mathrm{~kg}$ and $150 \mathrm{mg}$ in patients weighing $\geq 50 \mathrm{~kg}$ ) or high-dose secukinumab (75 mg in patients weighing $<25 \mathrm{~kg}, 150 \mathrm{mg}$ in patients weighing 25 to $<$ $50 \mathrm{~kg}$, and $300 \mathrm{mg}$ in patients weighing $\geq 50 \mathrm{~kg}$ ). Secukinumab was administered at weeks $0,1,2,3$, and 4 , then every 4 weeks. Historical placebo data from placebo-controlled trials in adult and pediatric patients with plaque psoriasis were used for the primary and key secondary endpoint analyses. The co-primary endpoints were the proportions of patients achieving PASI 75 and IGA 0/1 responses at week 12 for secukinumab compared with historical placebo [16].

\subsubsection{Clinical Response}

Secukinumab demonstrated efficacy in pediatric patients with moderate to severe plaque psoriasis [16]. At week 12, both low- and high-dose secukinumab were superior to historical placebo in terms of PASI 75, IGA 0/1, and PASI 90 response rates ( $p$-values not stated; Table 2 ); the estimated probability of a positive treatment effect over historical placebo was $1(100 \%)$ [16]. PASI 75 and IGA 0/1 response rates increased until week 24 (low-dose: 95.2 and 88.1\%; highdose: 95.2 and 92.9\%) [16] and were sustained up to week 52 (low-dose: 88.1 and 85.7\%; high-dose: 90.5 and 83.3\%) [19]. 
PASI 90/100 response rates at week 52 were $76.2 / 52.4 \%$ in the low-dose secukinumab group and $83.3 / 69.0 \%$ in the high-dose secukinumab group [19]. PASI 90 response rates were numerically higher with high- versus low-dose secukinumab at weeks 32, 48 and 52 (but not at week 40). Similarly, PASI 100 response rates were numerically higher with high- versus low-dose secukinumab from week 32 until week 52 [19]. The efficacy of secukinumab was consistent across bodyweight ( $<25 \mathrm{~kg}, 25$ to $<50 \mathrm{~kg}$, and $\geq 50 \mathrm{~kg}$ ) and age ( 6 to $<12$ years and 12 to $<18$ years) subgroups [20].

\subsubsection{Health-Related Quality of Life}

Secukinumab improved HR-QOL in pediatric patients with moderate to severe plaque psoriasis [18]. At week 12, $\geq 50 \%$ of patients treated with secukinumab achieved a CDLQI 0/1 response at week 12 (Table 2). These response rates further increased up to week 24 in the low-dose secukinumab group (70.7\%) and from week 24 to week 52 in the highdose secukinumab group (70.3\%). Consistently, the mean CDLQI total score decreased (improved) through week 24 in the low-dose group and through week 52 in the high-dose group. The mean absolute change from baseline in CDLQI total score at week 52 was -8.7 with low-dose secukinumab and -11.9 with high-dose secukinumab. Similarly, improvements in all individual domain scores (except personal relationships and treatment) were numerically higher with highversus low-dose secukinumab at week 52 [18].

\section{Tolerability of Secukinumab}

Secukinumab was generally well tolerated in pediatric patients with plaque psoriasis, with no new safety signals identified $[15,16,21]$. The safety profile in this population was consistent with that reported in adults with plaque psoriasis $[8,9]$.

In a pooled analysis of the phase III trials discussed in Sect. $2(n=198)$, the overall incidence of adverse events (AEs) up to week 52 was $74.5 \%$ with any low dose of secukinumab, $74.0 \%$ with any high dose of secukinumab, and $82.9 \%$ with etanercept [21]. Corresponding rates of serious AEs were 7.1, 6.0, and $12.2 \%$, respectively. The most common (incidence $\geq 10 \%$ ) AEs reported up to week 52 were nasopharyngitis $(23.5 \%$ with any low dose of secukinumab and $26.0 \%$ with any high dose of secukinumab vs $26.8 \%$ with etanercept) and headache (10.2 and 10.0 vs 9.8\%) [21].

In the severe plaque psoriasis trial, $3 \%$ of secukinumab recipients and $2 \%$ of etanercept recipients discontinued treatment due to AEs [15]. No deaths were reported and there were no new safety concerns with long-term secukinumab exposure (up to week 104) [17]. In the moderate to severe plaque psoriasis trial, AEs leading to treatment discontinuation occurred in $2 \%$ of patients [16].

\subsection{Adverse Events of Special Interest}

Treatment with secukinumab may increase [9], or has the potential to increase [8], the risk of infection. In the pooled analysis, infections and infestations occurred in 58\% of secukinumab recipients and $66 \%$ of etanercept recipients up to week 52 [21]. The incidence of infections and infestations did not differ based on age $(<12$ and $\geq 12$ years) or bodyweight ( $<25, \geq 25$ to $<50$, and $\geq 50 \mathrm{~kg}$ ). Up to week 52 , neutropenia occurred in $3 \%$ of secukinumab recipients and $2 \%$ of etanercept recipients, while Candida infections occurred in $2 \%$ of secukinumab recipients and $0 \%$ of etanercept recipients [21]. Caution is advised when considering the use of secukinumab in patients with chronic infections or a history of recurrent infection $[8,9]$. Before starting secukinumab, patients should be evaluated for tuberculosis infection [9], as secukinumab should not be given to patients with active tuberculosis $[8,9]$. Secukinumab should be discontinued in patients who develop a serious infection; close monitoring of these patients is also recommended $[8,9]$.

Cases of new or exacerbations of inflammatory bowel disease (IBD) have occurred in adult patients receiving secukinumab $[8,9]$. There were no reports of IBD in the severe plaque psoriasis trial [15]. One potential case of IBD in the moderate to severe plaque psoriasis trial was ruled out as mild, non-serious hemorrhagic diarrhea [16]. The use of secukinumab in patients with IBD is not recommended in the EU [8]. In the USA, caution is advised when prescribing secukinumab to patients with IBD [9].

In the pooled analysis, $4 \%$ of secukinumab injections (vs $10 \%$ of etanercept injections) were associated with injectionsite reactions (ISRs) up to week 52 [21]. All ISRs were mild in severity and most required no treatment [15].

There have been reports of hypersensitivity reactions (including anaphylaxis and urticaria) in patients receiving secukinumab $[8,9]$. In the pooled analysis, hypersensitivity reactions occurred in $10 \%$ of secukinumab recipients and $12 \%$ of etanercept recipients up to week 52 [21]. Secukinumab is contraindicated in patients with a previous hypersensitivity reaction to secukinumab $[8,9]$. Anaphylactic or other serious allergic reactions to secukinumab should be treated with appropriate therapy, and the drug should be discontinued $[8,9]$.

As with all therapeutic proteins, secukinumab has the potential for immunogenicity [9]. However, among pediatric patients treated with secukinumab in the severe plaque psoriasis trial, no anti-drug antibodies were detected [22]. 


\section{Dosage and Administration of Secukinumab}

Subcutaneous secukinumab is approved in the EU [8] and the USA [9] for the treatment of moderate to severe plaque psoriasis in pediatric patients aged $\geq 6$ years who are candidates for systemic therapy (or phototherapy [9]). It is available as a lyophilized powder in a vial for reconstitution or as a solution for injection in a prefilled syringe or Sensoready ${ }^{\circledR}$ pen $[8,9]$. The powder formulation should be administered by healthcare providers only, while the prefilled syringe or pen may be administered by an adult caregiver after proper training in subcutaneous injection technique $[8,9]$.

Secukinumab is administere at weeks $0,1,2,3$, and 4 , followed by maintenance dosing every 4 weeks $[8,9]$. Each injection should be administered at a newer anatomic location (e.g. thigh, abdomen, upper arm) than the previous injection, avoiding areas where the skin is tender, bruised, erythematous, indurated, or scaly. The recommended dose of secukinumab is $75 \mathrm{mg}$ in patients weighing $<50 \mathrm{~kg}$ and $150 \mathrm{mg}$ in patients weighing $\geq 50 \mathrm{~kg}[8,9]$. In the EU, the dose of secukinumab may be increased to $300 \mathrm{mg}$ in patients weighing $\geq 50 \mathrm{~kg}$ [8]. Discontinuation of secukinumab should be considered if patients do not respond after 16 weeks of treatment; patients with an initial partial response may subsequently improve with continued treatment beyond 16 weeks [8].

The concomitant administration of live vaccines with secukinumab is not recommended [8,9]. Patients should receive all age-appropriate immunizations prior to starting secukinumab. The efficacy and tolerability of secukinumab in pediatric patients aged $<6$ years has not been established $[8,9]$. Consult local prescribing information for details regarding contraindications, warnings and precautions, drug interactions, and use in special patient populations.

\section{Place of Secukinumab in the Management of Moderate to Severe Pediatric Plaque Psoriasis}

Secukinumab is a targeted biologic recently approved for use in children aged $\geq 6$ years and over with moderate to severe plaque psoriasis (Sect. 4). Joint American Academy of Dermatology and National Psoriasis Foundation guidelines for pediatric psoriasis published prior to the approval of secukinumab strongly recommend biologics as effective therapies for patients aged $\geq 4$ years (e.g. etanercept, adalimumab) and $\geq 12$ years (e.g. ustekinumab) with moderate to severe psoriasis [3]. Likewise, prior to the approval of secukinumab, European consensus-based guidelines for the treatment of psoriasis in children and adolescents recommend adalimumab as the only approved first-line treatment [23]. Etanercept, ustekinumab, ixekizumab, and secukinumab are among the second-line systemic agents that may be recommended or considered for the treatment of moderate to severe psoriasis in children and adolescents who have not sufficiently responded to at least one other systemic treatment or phototherapy, or who do not tolerate these treatments [23]. The National Institute for Health and Care Excellence recommends secukinumab as a treatment option for children and adolescents aged 6-17 years with severe plaque psoriasis who have not responded to other systemic treatments, or where these options are contraindicated or not tolerated [24].

Approval of secukinumab was based on the findings of two pivotal phase III trials, in which secukinumab provided fast and durable skin clearance in pediatric patients aged 6 to $<18$ years with moderate to severe plaque psoriasis (Sects. 2.1.1 and 2.2.1). The co-primary endpoints were met, with both low $(75-150 \mathrm{mg})$ and high $(75-300 \mathrm{mg})$ doses of secukinumab demonstrating significantly higher PASI 75 and IGA 0/1 response rates than placebo (Sects. 2.1.1 and 2.2.1). In the severe plaque psoriasis trial, secukinumab was associated with greater clinical improvement than etanercept (Sect. 2.1.1). Moreover, higher response rates were observed with high- versus low-dose secukinumab in patients weighing $\geq 50 \mathrm{~kg}$ (Sect. 2.1.1), confirming that patients in this subgroup who do not respond sufficiently to the lower dose may derive additional benefit from the higher dose $[8,15]$. Indeed, in the EU, the dose of secukinumab can be increased from 150 to $300 \mathrm{mg}$ in patients weighing $\geq 50 \mathrm{~kg}$ (Sect. 4).

The clinical efficacy of secukinumab observed during the first 12 weeks of treatment was sustained over the longer term, up to 52 weeks (Sect. 2.2) and 104 weeks (Sect. 2.1). Both trials are ongoing, with efficacy and tolerability data being collected up to 224 and 236 weeks, including 16 weeks of treatment-free follow-up [15, 19]. Final results of these trials will provide additional data and are awaited with interest.

Plaque psoriasis is a chronic, multisystem disease which can have a significant negative impact on QOL in terms of physical, emotional, social, and psychological function [3]. Treatment of pediatric psoriasis must take into account the unique psychosocial burden of the disease on patients and their families [6]. Alleviation of symptoms is an important goal in the management of psoriasis, which can be assessed not only in terms of improvement in skin lesions, but also in terms of improvement in HR-QOL [4]. In both phase III trials, secukinumab was associated with continuous improvement in HR-QOL (Sects. 2.1.2 and 2.2.2). Of note, up to two-thirds of secukinumab recipients reported that psoriasis had no impact on QOL at the end of maintenance treatment; 
this was maintained over the longer term, up to 104 weeks (Sect. 2.1.2).

The tolerability profile of secukinumab in pediatric patients with plaque psoriasis was consistent with that observed in adults with plaque psoriasis (Sect. 3). Secukinumab was generally well tolerated, with nasopharyngitis being the most commonly reported $\mathrm{AE}$ (Sect. 3 ). The rates of AEs of special interest with secukinumab were generally low (Sect. 3.1). It should be noted that secukinumab was associated with fewer ISRs than etanercept (Sect. 3.1). No new safety signals were identified through 52 and 104 weeks of treatment; longer-term follow-up is ongoing.

Targeted biologic agents offer several advantages over conventional systemic therapies, including less frequent dosing, enhanced efficacy, and reduced laboratory monitoring [6]. As with all of the biologic agents currently approved for pediatric plaque psoriasis, secukinumab is administered via subcutaneous injection (Sect. 4). After proper training, secukinumab may be administered by an adult caregiver using a pre-filled syringe or pen (Sect. 4). Unlike etanercept, which is administered once weekly, and adalimumab, which is administered every 2 weeks, maintenance doses of secukinumab (and ustekinumab and ixekizumab) are administered once every 4 weeks (Sect. 4). The convenience of less frequent dosing and the option of caregiver administration may result in better treatment adherence.

In conclusion, although longer-term data will more definitively place secukinumab in relation to other biologic agents in the management of pediatric plaque psoriasis, it is an effective and generally well-tolerated treatment for children and adolescents with moderate to severe plaque psoriasis, and represents a valuable new addition to the limited treatment options available for this population.

\section{Data Selection Secukinumab: 84 records identified}

Duplicates removed

Excluded during initial screening (e.g. press releases; news reports; not relevant drug/indication; preclinical study; reviews; case reports; not randomized trial)

Excluded during writing (e.g. reviews; duplicate data; small patient number; non-randomized/phase I/II trials)

Cited efficacy/tolerability articles

Search Strategy: EMBASE, MEDLINE and PubMed from 1946 to present. Clinical trial registries/databases and websites were also searched for relevant data. Key words were secukinumab, Cosentyx, pediatric, plaque psoriasis. Records were limited to those in English language. Searches last updated 27 September 2021
Supplementary Information The online version contains supplementary material available at https://doi.org/10.1007/s40272-021-00476-w.

Acknowledgements During the peer review process, the manufacturer of secukinumab was also offered an opportunity to review this article. Changes resulting from comments received were made on the basis of scientific and editorial merit.

\section{Declarations}

Funding The preparation of this review was not supported by any external funding.

Authorship and Conflict of interest Hannah Blair is a salaried employee of Adis International Ltd/Springer Nature, and declares no relevant conflicts of interest. All authors contributed to the review and are responsible for the article content.

Ethics approval, Consent to participate, Consent to publish, Availability of data and material, Code availability Not applicable.

Open Access This article is licensed under a Creative Commons Attribution-Noncommercial 4.0 International License, which permits any non-commercial use, sharing, adaptation, distribution and reproduction in any medium or format, as long as you give appropriate credit to the original author(s) and the source, provide a link to the Creative Commons license, and indicate if changes were made. The images or other third party material in this article are included in the article's Creative Commons license, unless indicated otherwise in a credit line to the material. If material is not included in the article's Creative Commons license and your intended use is not permitted by statutory regulation or exceeds the permitted use, you will need to obtain permission directly from the copyright holder. To view a copy of this license, visit http:// creativecommons.org/licenses/by-nc/4.0/.

\section{References}

1. Nogueira M, Paller AS, Torres T. Targeted therapy for pediatric psoriasis. Paediatr Drugs. 2021;23(3):203-12.

2. Wu JJ, Merola JF, Feldman SR, et al. Treatment of psoriasis with secukinumab in challenging patient scenarios: a review of the available evidence. Dermatol Ther. 2020;10(3):351-64.

3. Menter A, Cordoro KM, Davis DMR, et al. Joint American Academy of Dermatology-National Psoriasis Foundation guidelines of care for the management and treatment of psoriasis in pediatric patients. J Am Acad Dermatol. 2020;82(1):161-201.

4. Eisert L, Augustin M, Bach S, et al. S2k guidelines for the treatment of psoriasis in children and adolescents-short version part 1. J Dtsch Dermatol Ges. 2019;17(8):856-70.

5. Haulrig MB, Zachariae C, Skov L. Off-label treatments for pediatric psoriasis: lessons for the clinic. Psoriasis (Auckl). 2021;11:1-20.

6. Cordoro KM. Toward optimal care of the pediatric patient with psoriasis: the new AAD-NPF management guideline. J Psoriasis Psoriatic Arthritis. 2020;5(1):7-11.

7. Blauvelt A, Chiricozzi A. The immunologic role of IL-17 in psoriasis and psoriatic arthritis pathogenesis. Clin Rev Allergy Immunol. 2018;55(3):379-90.

8. European Medicines Agency. Secukinumab (Cosentyx $\left.{ }^{\circledR}\right)$ : EU summary of product characteristics. 2020. https://www.ema. europa.eu. Accessed 4 Oct 2021. 
9. Novartis. COSENTYX ${ }^{\circledR}$ (secukinumab): US prescribing information. 2021. https://www.accessdata.fda.gov. Accessed 4 Oct 2021.

10. Garnock-Jones KP. Secukinumab: a review in moderate to severe plaque psoriasis. Am J Clin Dermatol. 2015;16(4):323-30.

11. Blair HA. Secukinumab: a review in psoriatic arthritis. Drugs. 2021;81(4):483-94.

12. Shirley M, Scott LJ. Secukinumab: a review in psoriatic arthritis. Drugs. 2016;76(11):1135-45.

13. Blair HA. Secukinumab: a review in ankylosing spondylitis. Drugs. 2019;79(4):433-43.

14. Blair HA, Dhillon S. Secukinumab: a review in ankylosing spondylitis. Drugs. 2016;76(10):1023-30.

15. Bodemer C, Kaszuba A, Kingo K, et al. Secukinumab demonstrates high efficacy and a favourable safety profile in paediatric patients with severe chronic plaque psoriasis: 52-week results from a phase 3 double-blind randomized, controlled trial. J Eur Acad Dermatol Venereol. 2020;35(4):938-47.

16. Magnolo N, Kingo K, Laquer V, et al. A phase III open-label, randomized multicenter study to evaluate efficacy and safety of secukinumab in pediatric patients with moderate to severe plaque psoriasis: 24-week results. J Am Acad Dermatol. 2021. https://doi. org/10.1016/j.jaad.2021.08.066.

17. Krasowska D, Gambichler T, Cortes C, et al. Two-year efficacy and safety profile of secukinumab in pediatric patients with severe chronic plaque psoriasis [abstract no. SP09]. In: 14th World Congress of Pediatric Dermatology. 2021.

18. Beissert S, Ballona R, Bartonova J, et al. Secukinumab improves quality of life of paediatric patients with moderate-to-severe plaque psoriasis: 52-week results from a phase III, randomised study [abstract no. P080 plus poster]. Pediatr Dermatol. 2021;38(Suppl. 1):43.

19. Reich A, Magnolo N, Kingo K, et al. Secukinumab treatment demonstrated high efficacy and safety in paediatric patients with moderate-to-severe plaque psoriasis: 52-week results from a randomised trial [abstract no. P121 plus poster]. Pediatr Dermatol. 2021;38(Suppl. 1):57-8.

20. Szepietowski JC, Sayed ME, Ghislain P-D, et al. Secukinumab demonstrated consistent efficacy across age and weight subgroups in pediatric patients with psoriasis: analyses from two phase 3 pediatric studies [abstract no. 78 plus poster]. In: Society for Pediatric Dermatology. 2021.

21. Sticherling M, Nikkels AF, Hamza AM, et al. Pooled safety analysis from phase 3 studies of secukinumab in paediatric patients with moderate to severe plaque psoriasis [abstract no. P1420]. In: 30th European Academy of Dermatology and Venereology Congress. 2021.

22. Bodemer C, Kaszuba A, Kingo K, et al. Secukinumab efficacy and safety profile in pediatric patients with severe chronic plaque psoriasis up to one year [abstract no. 25482 plus poster]. In: American Academy of Dermatology Virtual Meeting Experience. 2021.

23. Eisert L, Augustin M, Bach S, et al. S2k guidelines for the treatment of psoriasis in children and adolescents-short version part 2. J Dtsch Dermatol Ges. 2019;17(9):959-73.

24. National Institute for Health and Care Excellence. Secukinumab for treating moderate to severe plaque psoriasis in children and young people. 2021. https://www.nice.org.uk. Accessed 4 Oct 2021. 